\title{
Metastatic Malignant Chondroid Syringoma: A Rare Case Scenario
}

\author{
Mahalaxmi Aal ${ }^{1}$, Vijay Bhaskar Lakshman ${ }^{1}$, Pradeep Kumar Karumanchi ${ }^{1}$, Gopinath Kodaganur Srinivas ${ }^{2}$, \\ Amarendra Shankarappa $^{2}$, Bindiya Vijayan ${ }^{1}$ \\ Departments of ${ }^{1}$ Radiation Oncology and ${ }^{2}$ Surgical Oncology, Health Care Global Enterprises Ltd, Bengaluru, Karnataka, India.
}

\section{Corresponding Author:}

Dr. Mahalaxmi Aal

Email: mahalakshmi.aall@gmail.com

This is an Open Access article distributed under the terms of the Creative Commons Attribution License (creativecommons.org/ licenses/by/3.0).

Received : June 15, 2019

Accepted : December 4, 2019

Published : January 30, 2020

\begin{abstract}
Background: Malignant chondroid syringoma is one of the rarest clinical entities. It is a mixed cutaneous tumor with propensity for local invasion resulting in multiple local recurrences. Malignant chondroid syringoma has female predilection common in extremities. We are presenting this case in view of its rare presentation among the population, and unique clinical course. Case Report: A 38-year-old lady, known case of malignant chondroid syringoma of the left hand was treated for multiple local recurrences with excision, local radiation therapy and chemotherapy. She developed distant visceral metastasis over a span of 20 years after the primary diagnosis. Conclusion: Malignant chondroid syringoma has potential for both local recurrence and distant metastasis over a prolonged clinical course with on and off remission period. Because of its longer clinical course patients are recommended to be on regular follow up.
\end{abstract}

Keywords: Computed Tomography, Biopsy, Mastectomy, Microcystic adnexal carcinoma, Skin Neoplasms.

\section{Introduction}

Malignant chondroid syringoma (MCS), an eccrine sweat gland neoplasm is one of the rarest presentations with as few as 50 cases have been reported worldwide till date [1]. MCS presents in trunk and extremities with invasive nature and frequent recurrences, unlike its benign counterpart which presents in head and neck region [2,3]. Here we present a rare case of metastatic malignant chondroid syringoma with a peculiar pattern of spread, treated earlier for multiple recurrences followed by development of distant metastases.

\section{Case Report}

A 38-year-old lady, proven case of recurrent malignant chondroid syringoma presented with the swelling in the left hand (first web space). This swelling first appeared at the age of 18 years. She underwent excision for the same after 10 years of appearance of the lesion in view of pain and increasing size of the lesion. After the first excision, in a span of 3 years she had recurrence at the same primary site and underwent repeat surgical excision. Post-operative histopathological diagnosis for both excision procedures was unknown. Thereafter, new recurrences were noted at the primary site (first web space of the left hand) and left axilla after 2 years. She was treated with excision of the left-hand lesion along with axillary clearance. Post-operative histopathology revealed microcystic adnexal carcinoma - chondroid syringoma with one axillary lymph node involved and Bcl-2 was positive. She received adjuvant external beam radiotherapy (EBRT) to a dose of $60 \mathrm{~Gy}$ in 30 fractions to the left hand primary recurrent site.

She was on follow up, and after a diseasefree interval of 2 years she developed recurrence in the left axilla and breast. She received 4 cycles of chemotherapy with three weekly paclitaxel 
and carboplatin, with no clinical and radiological response. Hence, she underwent lumpectomy and received adjuvant external beam radiation therapy to a dose of 60 Gy in 30 fractions to the left axilla and breast. Six months later, she developed two recurrent lesions in the left breast and in the first web space of the left hand. Wide local excision, excision of nipple areolar complex and re-excision of the left thenar space of the hand was done. Post-operative histopathology revealed recurrent malignant chondroid syringoma. Immunohistochemistry showed Ki-67 index $60 \%$, diffusely positive for EMA, p63 and focally positive for PR receptor, ER/Her2neu, GATA-3, ER and GCDFP were negative.

Patient on follow up developed pain in the right lower limb and upper abdominal discomfort. She was evaluated with whole body PET CT scan which showed wide spread metastases to liver, left kidney, pancreas, lungs, head \& shaft of right femur and multiple paraaortic lymph nodes with extensive tumor thrombus in the above-mentioned visceral organs. Ultrasound guided core needle biopsy of liver was consistent with metastatic skin adnexal tumor - chondroid syringoma to the liver. Immunohistochemistry of tumor cells expressed EMA and was negative for S-100, Desmin and CD34.

She was treated with palliative radiation therapy to the skeletal lesion in the right femur in view of pain. After the diagnosis of distant metastases, patient survived for 4 months with supportive care. Overall clinical course of the patient was more than 22 years with multiple disease-free intervals till the development of metastases.

\section{Discussion}

Malignant chondroid syringoma was first described by Hirsch and Helwig in 1961 [2,3]. It is an extremely rare tumor of sweat glands with no evidence of a specific risk factor. Trauma has been suggested as a possible predisposing factor for the development of both malignant and benign chondroid syringoma [3]. MCS is included in myoepithelial tumors of the skin and has an epithelial component with eccrine differentiation [1,3]. It is preferentially seen at extremities and trunk. Epidemiologically, MCS have a female predilection while their benign counterparts are common in men $[2,3]$. The spread can be lymphatic, or blood borne. The most common sites for distant metastases are lungs, bones and brain $[1,3]$, characterized by a faster growth and size significantly greater than that of benign variant [3].

Pathological diagnosis forms an integral component in terms of management in malignant chondroid syringoma. The very first histopathological diagnosis is crucial to plan further treatment in terms of re-surgery with good margins if only excision is done upfront. Detailed histopathological examination by an oncopathology expert along with immunohistochemistry aids in early diagnosis of the disease. Role of genomics at the earliest might be considered as well in MCS. The available literature suggests that the clinical course of MCS is deemed unpredictable. MCS behaves more aggressively than other skin carcinomas with a series of local recurrences over a span of many years [2]. Early wide local excision with adequate margins appears to be the most reliable treatment. Adjuvant radiotherapy is useful in reducing local recurrence to a limited extent. There is no evidence of effectiveness of chemotherapy [3].

In this case, patient had extensive local recurrences in the web space of left hand followed by left axilla, left breast and eventually with distant metastases to various internal organs over a span of more than 22 years, treated with multiple surgical excisions in the area of recurrences followed by adjuvant radiation therapy. Chemotherapy didn't prove to be effective, as there was progression of the disease. Distant metastasis was noted at a very late point of time. We observed that, so far this is 
the only case being reported with a large interval of time between the initial diagnosis and development of visceral metastases. Default in regular follow up is a missed opportunity to deliver an effective treatment to curtail further progression of the disease.

\section{Conclusion}

MCS is a rare clinical entity with a potential for recurrences in multiple sites and distant metastases over a span of many years [4]. Patient should be accurately diagnosed upfront with good pathological evaluation, which should include IHC and genomics and imaging plays an important role to know the spread of the disease either local or distant metastases [1]. In view of aggressive nature of the disease spreading in a sequence of local and regional pattern before development of extensive metastases, we suggest contemplating the role of surgical intervention at the regional lymph nodes to be explored at the earliest. Patient education regarding regular follow-ups for recurrences are critical which makes it feasible for surgical excision with radical intent [4] and helps in facilitating long term survival.
Contributors: MA, VBL: manuscript writing, and literature review and references; PKK, GKS, BV: critical inputs into the manuscript and patient management; AS: literature review, discussion and surgical management. MA will act as a study guarantor. All authors approved the final version of this manuscript and are responsible for all aspects of the study.

Funding: None; Competing interests: None stated.

\section{References}

1. Garcia AM, Atun JM, Fernando G. Multifocal, recurrent malignant chondroid syringoma with visceral metastases: A case report and literature review. International Journal ofCancer Therapy and Oncology. 2016;4.4119.10.14319/ ijcto.41.19.

2. Malik R, Saxena A, Kamath N. A rare case of malignant chondroid syringoma of scalp. Indian Dermatology Online Journal. 2013;4(3):236-238.

3. Ka S, Gnangnon F, Diouf D, Dieng MM, Thiam J, Gaye $\mathrm{M}$, et al. Malignant chondroid syringoma in a West African cancer institute. A case report. International Journal of Surgery Case Reports. 2016;25:137-138.

4. Kothiya M, Mittal N, Kumar R, Kane S. Malignant chondroid syringoma of thigh with late metastasis to lung. A very rare case report. Indian Journal of Pathology and Microbiology. 2017;60(3):418. 